\title{
BIOLOGÍA Y COMPORTAMIENTO DE Amauromyza maculosa (MALLOCH, 1913) (DIPTERA: AGROMYZIDAE) EN ALCACHOFA (Cynara scolimus L.) Y GIRASOL (Helianthus annus L.)
}

\section{BIOLOGY AND BEHAVIOR OF Amauromyza maculosa (MALLOCH, 1913) (DIPTERA: AGROMYZIDAE) IN ARTICHOKE (Cynara scolimus L.) AND SUNFLOWER (Helianthus annus L.)}

\author{
Lucy Enríquez ${ }^{1}$, Jorge Castillo² y Susana Rodríguez ${ }^{3}$
}

\begin{abstract}
Resumen
En alcachofa, el ciclo biológico de Amauromyza maculosa (Malloch) duró 22.6 días. El huevo es endofítico y el período de incubación fue de 2 días; el periodo larval del primer y segundo estadío fue de 2 días cada uno y el tercer estadío de 3 días; la pupa tuvo una duración de 11 a 14 días. El período de oviposición fue de 38.8 días, la capacidad de oviposición fue de 326.6 huevos y la proporción sexual (M:H) fue de 1.1: 1.5. En girasol, el ciclo biológico duró 19.8 días. El huevo, la larva del primer y segundo estadío tuvieron una duración de 2 días cada uno, en dos generaciones, y de 1 día en la tercera generación; y el tercer estadío larval duró 3 días en la primera y segunda generación y 2.5 días en la tercera generación. La pupa tuvo una duración de 10 a 12 días. El período de oviposición fue de 35.7 días, la capacidad de oviposición fue de 345.3 huevos y la proporción sexual (M:H) fue de 1: 1.5 .

Palabras clave: Amauromyza maculosa, ciclo biológico, alcachofa, Cynara scolimus L., girasol Helianthus annus L.
\end{abstract}

\begin{abstract}
In artichoke, the life cycle of Amauromyza maculosa (Malloch) lasted 22.6 days. The egg is endophytic and the incubation period was 2 days; the larval period of the first and second stage was 2 days each and the third stage of 3 days; pupa lasted 11 to 14 days. Oviposition period was 38.8 days, the capacity was 326.6 oviposition eggs and sex ratio (M: F) was 1.1: 1.5. In sunflower, the life cycle takes 19.8 days. The egg, larva first and second stage lasted two days each one, in two generations, and 1 day in the third generation; and third larval stage lasted 3 days in the first and second generation and 2.5 days in the third generation. The pupa lasted 10 to 12 days. Oviposition period was 35.7 days, the oviposition eggs capacity was 345.3 and sex ratio (M: F) was 1: 1.5 .
\end{abstract}

Key words: Amauromyza maculosa, life cycle, artichoke, Cynara scolimus L., sunflower, Helianthus annus L.

\section{Introducción.}

En el Perú en los últimos años se ha incrementado el área para el cultivo de alcachofa (Cynara scolymus L.) por su gran potencial exportador y porque en nuestro medio es posible obtener cosechas durante casi todo el año. (Robles, 2000). Las principales plagas que se presentan en este cultivo son: Copitarsia sp., Spodoptera sp., Agrotis ípsilon (Hufnagel), Feltia sp., Macrosiphum sp., Aphis fabae (Scopoli), Bemisia sp., Liriomyza huidobrensis (Blanchard), Amauromyza sp., Argyrotaenia sp., Anomala sp., Cyclocephala sp., Lygirus sp., Vaginulus sp., Limax sp. y Agriolimax sp. (Castillo, 2006), según observaciones descritas en las larvas de Amauromyza maculosa, "minador lagunar de hojas”, se producen minas en forma de laguna, formadas principalmente en la porción apical de las hojas, apareciendo como una ampolla, (Raven, 1993).
El daño causado por A. maculosa puede propiciar la presencia de hongos, tal como menciona Cisneros (1995), que algunos patógenos aprovechan las heridas producidas por los insectos para atacar las plantas.

Amauromyza maculosa (Malloch), es abundante en Norteamérica, en la actualidad, en el Perú, $A$. maculosa no ha sido muy estudiada. En base a estas consideraciones, el presente trabajo de investigación tuvo como objetivos determinar el ciclo biológico y el comportamiento en laboratorio de Amauromyza maculosa, en alcachofa y girasol.

\section{Materiales y métodos.}

El presente trabajo se llevó a cabo en el Departamento de Entomología de la Universidad Nacional Agraria La Molina, en el distrito La Molina, provincia y departamento de Lima - Perú, bajo 
condiciones controladas de laboratorio. El desarrollo de A. maculosa (Blanchard) en la primera generación tuvo una temperatura de $24 \pm 1^{\circ} \mathrm{C}$ y $88 \pm 2 \%$ de humedad relativa, en la segunda generación la temperatura de $25 \pm 1^{\circ} \mathrm{C}$ y $91 \pm 2 \%$ de humedad relativa y tercera generación a una temperatura de $26 \pm 1^{\circ} \mathrm{C}$ y $90 \pm 2 \%$ de humedad relativa. Para realizar la investigación se sembraron plantas de alcachofa y girasol en macetas de plástico en siembras escalonadas para asegurar la disponibilidad de plantas en el momento oportuno.

Para disponer de A. maculosa se colectaron hojas de alcachofa que presentaban "minas lagunares", daño típico de su presencia, obtenidas de los campos de alcachofa del fundo agrícola Agromalambo ubicado en el distrito de Potao de la provincia de Barranca. Las hojas fueron trasladadas al laboratorio y al día siguiente, las hojas fueron acondicionadas en jaulas de recuperación de $50 \mathrm{~cm}$. x $33 \mathrm{~cm}$. x $18 \mathrm{~cm}$. Después de cuatro días se procedió a recuperar puparios de $A$. maculosa los que fueron colocados en placas Petri conteniendo arena lavada y desinfectada. Estas placas con puparios se adecuaron dentro de una jaula de vidrio de $15 \mathrm{~cm} \times 15 \mathrm{~cm}$ x $15 \mathrm{~cm}$ cubierta con tela de organza hasta la emergencia de los adultos, los cuales diariamente fueron trasladados a una jaula de dos mangas de madera y vidrio de $76.5 \mathrm{~cm} \mathrm{x} 41 \mathrm{~cm} \mathrm{x} 52$ $\mathrm{cm}$. Los adultos colectados en campo se remitieron al Servicio Nacional de Sanidad Agraria (SENASA) Lima, los que fueron identificados como Amauromyza maculosa (Malloch).

La crianza de la primera generación se realizó con adultos recuperados de campo, la segunda con adultos provenientes de la primera generación y la tercera con adultos obtenidos de la segunda generación, teniendo como hospedero a plántulas de alcachofa y girasol por separado. Al comenzar la crianza $A$. maculosa se colocaron dos macetas y 30 parejas de adultos sexados en el interior de una jaula de dos mangas, las macetas fueron revisadas y cambiadas diariamente para observar y contabilizar las posturas. En las hojas fueron marcadas con plumón indeleble 30 posturas y se realizó el seguimiento desde huevo hasta adulto; en cada hoja se dejaron sólo 2 posturas, las restantes fueron utilizadas para la crianza masal de la cual se tomaron los datos de mediciones y de comportamiento de los diferentes estados biológicos. Este procedimiento se aplicó en las tres generaciones de $A$. maculosa en alcachofa y girasol.

Se estudiaron 30 huevos marcados, registrándose la fecha que fueron ovipuestos en las hojas de su hospedero y la fecha de eclosión de los mismos. El tiempo transcurrido entre la oviposición y la eclosión permitió determinar el periodo de incubación. Con el estereoscopio se observaron 30 larvas recién emergidas, considerándose a estas como larvas de primer estadio. El tamaño y la forma del diseño de ganchos mandibulares, sirvieron para determinar el estadio larval correspondiente. Se anotó el tiempo en días que permanecieron en cada estadio larval. De una crianza paralela a la del estudio de biología, se tomaron 30 especímenes de cada estadio, los cuales se conservaron en alcohol de $70^{\circ}$. Se procedió a la decoloración de las larvas para realizar la medición de los ganchos mandibulares. Las larvas fueron retiradas del alcohol de $70^{\circ}$ y se enjuagaron 3 veces con agua destilada, agregándose posteriormente $3 \mathrm{ml}$ de $\mathrm{KOH}$ al $10 \%$, se colocaron en baño maría por espacio de 20 minutos y se lavaron con agua destilada 3 veces más, se realizó el montaje de las larvas en un medio a base de glicerina y laminillas porta y cubre objeto. Con la ayuda de un estereoscopio con ocular micrométrico se procedió a realizar las mediciones.

Puparios en número de 30 fueron acondicionados en placas Petri, las cuales estaban provistas de arena ligeramente humedecida con agua. Para determinar la duración del periodo pupal se consideró el tiempo transcurrido desde la formación del pupario hasta la emergencia del adulto. Se observó el cambio de coloración del pupario.

Para la determinación del sexo de los adultos obtenidos en cada generación se procedió a colocar los adultos emergidos el mismo día en una placa Petri a contra luz y con la ayuda de una lupa de 20x se observó el ovipositor y los ojos dicópticos de la hembra, y los ojos holópticos en el macho. Se acondicionaron adultos en una jaula sobre una maceta con una planta hospedera, 2 machos y 1 una hembra. La jaula estaba constituida por un taper de plástico transparente con dos ventanas laterales y una superior de organza, sin fondo. Esta jaula estaba asegurada con cinta adhesiva de papel. Las plantas fueron revisadas a diario para contar los huevos puestos y determinar la capacidad de oviposición. También se determinó el período de pre-oviposición, oviposición, postoviposición y la longevidad de adultos macho y hembra. Sobre las hojas se colocaron pequeñas gotas de miel para la alimentación de los adultos. Para esta parte del estudio fue necesario emplear cinco repeticiones en cada generación y hospedero.

Para el diseño estadístico se compararon los promedios del período de incubación, desarrollo larval, período pupal, longevidad de adultos macho y hembras, período de preoviposición, oviposición y capacidad de oviposición, utilizando el modelo estadístico no paramétrico de Kruskall-Wallis, con un nivel de significación de 0.05 (Siegel, 1982). El diseño se aplicó en las tres generaciones y en ambos tipos de alimentación, alcachofa y girasol.

\section{Resultados y discusión. \\ Huevo.}

El adulto hembra colocó sus huevos individualmente en el haz de las hojas maduras bajo la epidermis, uno cerca del otro y en el borde de la hoja. El huevo es ovalado no presentando ornamentaciones, de color blanco claro recién colocado, cambiando a 
blanco cremoso próximo a la emergencia. En alcachofa midió $0.34 \mathrm{~mm}, 0.33 \mathrm{~mm}$ y $0.31 \mathrm{~mm}$ en las tres generaciones; en girasol midió $0.33 \mathrm{~mm}, 0.32 \mathrm{~mm}$ y $0.31 \mathrm{~mm}$ en las tres generaciones respectivamente. Los huevos que fueron colocados en hojas jóvenes pasaron por el proceso de extrusión del huevo por el tejido en crecimiento, esto mismo ocurre para $L$. huidobrensis (Blanchard) (Lizárraga, 1989). En alcachofa el promedio del período de incubación fue de 2 días con un rango de 1.5 a 2.0 días en cada una de las tres generaciones estudiadas. Entre las generaciones no se encontraron diferencias significativas con un nivel de significancia de 0.05. En girasol, el promedio del periodo de incubación fue de 2 días con un rango de 1.5 a 2.0 días en la primera y segunda generación, sin embargo, en la tercera generación fue en promedio de 1 día con un rango de 1 a 2 días por condiciones no específicas. Entre la primera y segunda generación con respecto a tercera generación, se encontraron diferencias significativas con un nivel de significancia de 0.05 . Estos resultados se aproximan numéricamente a lo encontrado por Prando \& Da Cruz (1986) en L. huidobrensis en condiciones de similares de temperatura y humedad relativa. Ver tabla 5.

Larva.

En cuanto a su biología, la larva pasó por tres estadíos, diferenciándose por la dimensión del gancho mandibular, que en promedio midió 0.017, 0.025 y 0.033 milímetros respectivamente, tanto en alcachofa como en girasol. En alcachofa, el primer y segundo estadio larval desarrollado en la primera generación, fue en promedio de 2 días con un rango de 1.5 a 2.0 días por cada estadío larval y en cada una de las generaciones. En el tercer estadío larval desarrollado en la primera generación, fue en promedio de 3 días con un rango de 3.0 a 4.0 días en cada una de las generaciones. No se encontraron diferencias significativas, con un nivel de significancia de 0.05, en la primera, segunda y tercera generación del primer estadío, el segundo estadío y tercer estadío. En girasol el primer y segundo estadío larval, fue en promedio de 2 días con un rango de 1.5 a 2.0 días en cada estadío larval en las dos generaciones, sin embargo, en la tercera generación, fue en promedio de 1 día con un rango de 1.0 a 2.0 días. Se encontraron diferencias significativas en la primera y segunda generación con respecto a la tercera generación en el primer y el segundo estadíos, con un nivel de significancia de 0.05 .

En girasol el tercer estadio larval, fue en promedio de 3 días con un rango de 3.0 a 4.0 días, sin embargo, en la tercera generación, fue en promedio de 2.5 días con un rango de 2.0 a 3.0 días. Encontrándose diferencias significativas entre la primera y segunda generación con respecto a la tercera generación del tercer estadío larval, con un nivel de significancia de 0.05. De acuerdo a los resultados, el periodo larval duró en promedio 7 días con un rango de 6 a 8 días en alcachofa y girasol, sin embargo, en la tercera generación en girasol duró en promedio 4.5 días con un rango de 4.5 a 6 días por condiciones no específicas; estos resultados se aproximan numéricamente a lo encontrado por Herrera (1963), Jara \& Alvites (1980) y Guzmán \& Vergara (1996) en L. huidobrensis bajo condiciones de similares de temperatura y humedad relativa.

De acuerdo al comportamiento observado, la larva de primer estadío realizó una mina delgada casi desapercibida; a partir del segundo estadío se llegó a juntar con otras larvas que se encontraban próximas observándose una mina lagunar pequeña que a medida que se alimentaban del parénquima de la hoja y se desarrollaban las larvas, la mina iba creciendo, el tercer estadío realizó un corte en la epidermis de la hoja saliendo de la mina y cayendo al suelo en donde se logró ocultar de la luz y permaneció hasta endurecer la epidermis y formarse el pupario. En cuanto al número de larvas encontrado por mina fue de 9 a 63 individuos observado en la crianza masal. En alcachofa, se encontraron más de 40 larvas por hoja y al no tener suficiente alimento atravesaron las nervaduras secundarias que son delgadas; en girasol, se observó que las larvas se alimentaron del peciolo de la hoja para completar su desarrollo, pero no llegaron a empupar por falta de espacio y de capacidad para romper ese tejido, quedando en el interior una pupa con aspecto achatado.

Pupa.

La pupa se encuentra protegida por un pupario que es de forma cilíndrica y de color amarillo cremoso recién formado cambiando su tonalidad a marrón oscuro próximo a la emergencia del adulto. Las pupas generadas a partir de larvas alimentadas con hojas de alcachofa midieron en promedio $2.81 \mathrm{~mm}, 2.41 \mathrm{~mm}$ y $2.32 \mathrm{~mm}$ de longitud en la primera, segunda y tercera generación respectivamente y aquellas alimentadas con hojas de girasol midieron en promedio $2.77 \mathrm{~mm}$, $2.38 \mathrm{~mm}$ y $2.22 \mathrm{~mm}$ de longitud en la primera, segunda y tercera generación respectivamente. A nivel de campo, las pupas se encontraron mayormente en el suelo hasta una profundidad de $3 \mathrm{~cm}$ entre las hojas caídas y secas muy cerca de la planta. También se observaron en el interior de la mina lagunar. A nivel de laboratorio en girasol, se encontraron altas poblaciones de pupas en el pecíolo de la hoja y tallo de la planta, pero de ellas no llegó a emerger ningún adulto.

El período pupal en alcachofa fue en promedio 14, 13 y 11 días, en cada generación. Entre la primera y segunda generación con respecto a la tercera generación, se encontraron diferencias significativas, con un nivel de significancia de 0.05. En girasol, el desarrollo pupal fue de 12, 11 y 10 días en cada generación. No se encontraron diferencias significativas en las tres generaciones, con un nivel de 
significancia de 0.05. Estos resultados se aproximan numéricamente a lo encontrado por Herrera (1963), Jara \& Alvites (1980), Palacios \& Cisneros (1980) y Lizárraga (1989) en estudios realizados en $L$. huidobrensis bajo condiciones similares de temperatura $\mathrm{y}$ humedad relativa.

Adulto.

El adulto presentó la mácula blanquecina en el halter, característica de A. maculosa, según Korytkowski (1982) y el informe de identificación del centro de Diagnóstico de Sanidad Vegetal Nro 1238-2007-AGSENASA-OCDP-UCDSV. Al emerger el adulto rompió el pupario por el extremo cefálico por presión del ptilinium, el cual es un órgano eversible ubicado debajo de la sutura frontal. Al momento de la emergencia del adulto, salió la cabeza y el primer par de patas, con las cuales se ayudó a salir de la exuvia pupal, realizando movimientos con la cabeza y las patas medias y posteriores hacia atrás, el primer par de patas le sirven de apoyo. El proceso de emergencia duró de 4 a 6 minutos; generalmente los adultos hembras emergieron primero; posteriormente lo hicieron los machos. La hembra se diferenció del macho por presentar pseudo ovipositor y ser de mayor tamaño, de la misma manera que lo observado por Lizárraga (1989) para L. huidobrensis. En adultos que emergen de larvas alimentadas con alcachofa, las hembras midieron de $2.14 \mathrm{~mm}$ a $2.42 \mathrm{~mm} \mathrm{y}$ los machos $1.60 \mathrm{~mm}$ a $2.01 \mathrm{~mm}$ en tanto que las hembras que se alimentaron de girasol midieron de $2.04 \mathrm{~mm}$ a $2.44 \mathrm{~mm}$ y los machos de $1.66 \mathrm{a} 1.99 \mathrm{~mm}$. Al emerger el adulto su cuerpo es de color gris, demorando 40 a 50 minutos en extender las alas y 25 a 30 minutos después su cuerpo comienza a tomar una coloración negro brillante, durante este proceso es poco activo realizando solamente saltos y vuelos cortos. La emergencia de los adultos ocurrió en horas de la mañana entre las 6 y las 11 a.m., observándose que la mayor emergencia fue alrededor de las 10 a.m. El apareamiento se realizó después de 2 a 5 horas de la emergencia, demorando la cópula de 3 a 8 minutos. Posteriormente la hembra realizó dos tipos de picadura con su pseudo-ovipositor: la picadura de oviposición, en la cual la hembra perforó la epidermis foliar y dejó el huevo; y la picadura de alimentación, que es superficial y le sirve para alimentarse de las exudaciones foliares. Cuando la hembra se retira cede el espacio al macho para que se alimente. El adulto tiene vuelo activo y es muy vivaz, pero cuando se encontró próximo a morir disminuyó su alimentación y solamente caminó en la superficie cercana a la planta.

Oviposición.

El periodo de pre-oviposición en alcachofa fue de 1.0, 1.2 y 1.1 días y en girasol fue de 1.1, 1.0 y 0.5 días en la primera, segunda y tercera
Tabla 1. Duración en días del periodo de pre-oviposición, oviposición y capacidad de oviposición de hembras de Amauromyza maculosa Malloch (Dip.: Agromyzidae) en alcachofa, en laboratorio y en tres generaciones. La Molina, Lima-Perú, 2006-2007.

\begin{tabular}{|c|c|c|c|c|}
\hline \multirow[t]{2}{*}{ eneración } & \multirow[t]{2}{*}{$\begin{array}{l}\text { Pre- } \\
\text { oviposición } \\
\text { (días) }\end{array}$} & \multirow[t]{2}{*}{$\begin{array}{l}\text { Oviposición } \\
\text { (días) }\end{array}$} & \multicolumn{2}{|c|}{$\begin{array}{c}\text { Capacidad de } \\
\text { oviposición } \\
\text { (Nro. de huevos por } \\
\text { hembra) }\end{array}$} \\
\hline & & & Diario & Total \\
\hline & 1.0 & 40.8 & 7.4 & 332.0 \\
\hline & 1.2 & 38.6 & 8.3 & 324.4 \\
\hline & 1.1 & 37.0 & 8.9 & 323.4 \\
\hline
\end{tabular}

generación, respectivamente (Tablas 1 y 2). El periodo de pre-oviposición fue menor en girasol que en alcachofa y difieren a lo señalado por Lizárraga (1989) respecto a $L$. huidobrensis en condiciones similares de temperatura y humedad relativa. El periodo de oviposición en alcachofa fue de 40.8, 38.6 y 37.0 días y en girasol fue de 38.4, 39.4 y 29.4 días en la primera, segunda y tercera generación respectivamente (Tablas 1 y 2). De los resultados, el periodo de oviposición fue mayor que el registrado por Lizárraga (1989) en L. huidobrensis en condiciones similares de temperatura y humedad relativa. La capacidad de oviposición en alcachofa fue de 332.0, 324.4 y 323.4 días y en girasol fue de 356, 354.4 y 325.6 días en la primera, segunda y tercera generación respectivamente (Tablas 1 y 2). De los resultados, el periodo de oviposición fue mayor que el registrado por Lizárraga (1989) en L. huidobrensis en condiciones similares de temperatura y humedad relativa. Así mismo, se registró un periodo de postoviposición de cero días en todas las generaciones.

Longevidad.

La longevidad de la hembra en alcachofa fue de 41 días en la primera y tercera generación y de 39 días en la segunda generación, en girasol fue de 39 días en la primera y segunda generación y de 36 días en la tercera generación. La longevidad del macho en alcachofa fue de 32, 31, 30 días en la primera, segunda y tercera generación respectivamente, en girasol fue de 27, 25 y 21 días en la primera, segunda y tercera generación respectivamente. La longevidad en hembras fue mayor que en los machos en ambos

Tabla 2. Duración en días del periodo de pre-oviposición, oviposición y capacidad de oviposición de hembras de Amauromyza maculosa Malloch (Dip.: Agromyzidae) en girasol en laboratorio y en tres generaciones. La Molina, Lima-Perú, 2006-2007.

\begin{tabular}{lcccc}
\hline Generación & $\begin{array}{c}\text { Pre- } \\
\text { oviposición } \\
\text { (días) }\end{array}$ & $\begin{array}{c}\text { Oviposición } \\
\text { (días) }\end{array}$ & $\begin{array}{c}\text { Capacidad de } \\
\text { oviposición } \\
\text { (Nro. de huevos por } \\
\text { hembra) }\end{array}$ \\
\cline { 3 - 5 } & & & Diario & Total \\
\hline GI & 1.1 & 38.4 & 9.4 & 356.6 \\
GII & 1.0 & 39.4 & 9.5 & 354.4 \\
GIII & 0.5 & 29.4 & 8.8 & 325.6 \\
\hline
\end{tabular}


Tabla 3. Proporción de sexos de Amauromyza maculosa Malloch (Dip.: Agromyzidae) en alcachofa bajo condiciones de laboratorio en tres generaciones. La Molina, Lima-Perú, 2006-2007.

\begin{tabular}{lcccccc}
\hline \multirow{2}{*}{ Generación } & \multirow{2}{*}{$\begin{array}{c}N^{\circ} \text { de } \\
\text { individuos }\end{array}$} & \multicolumn{2}{c}{ Macho (M) } & \multicolumn{2}{c}{ Hembra (H) } & Proporción \\
\cline { 3 - 6 } & 25 & 11 & 44 & 14 & 56 & $1.3: 1.0$ \\
\hline GI & 25 & 9 & 36 & 16 & 64 & $1.7: 1.0$ \\
GII & 25 & 10 & 40 & 15 & 60 & $1.5: 1.0$ \\
GIII & & &
\end{tabular}

hospederos en las tres generaciones. Comparando los dos hospederos, los adultos que se alimentaron con hojas de alcachofa son de más grandes y más longevos que los alimentados con girasol. Se encontraron diferencias significativas en la longevidad de hembras $\mathrm{y}$ de machos en las tres generaciones, con un nivel de significancia de 0.05 .

Proporción sexual.

La proporción sexual $(\mathrm{M}: \mathrm{H})$ en alcachofa fue de 1.3:1.0, 1.7:1.0 y 1.5: 1.0 en la primera, segunda $\mathrm{y}$ tercera generación, respectivamente y en girasol fue de 1.5: 1.0 en las tres generaciones (Tablas 3 y 4). Estos resultados son muy semejantes a la proporción encontrada por Palacios \& Cisneros (1980) y Lizárraga (1989) en similares condiciones de temperatura y humedad relativa. Así mismo, de 100 pupas obtenidas de campo con cultivo de alcachofa, $52 \%$ son

hembras y $28 \%$ machos.

Parasitismo.

Tabla 5. Promedio en días de los estados de desarrollo de Amauromyza maculosa Malloch (Dip.: Agromyzidae) en alcachofa bajo condiciones de laboratorio en tres generaciones. La De las pupas Molina, Lima-Perú. 2006-2007.

\begin{tabular}{|c|c|c|c|c|c|c|c|c|c|c|}
\hline \multirow{2}{*}{ obtenidas de } & \multirow{2}{*}{\multicolumn{2}{|c|}{ Generación }} & \multirow{2}{*}{ Huevo } & \multicolumn{3}{|c|}{ Estadíos Larvales } & \multirow{2}{*}{ Pupa } & \multicolumn{2}{|c|}{ Longevidad } & \multirow{2}{*}{$\begin{array}{c}\text { Ciclo } \\
\text { Biológico }\end{array}$} \\
\hline & & & & 1 & 2 & 3 & & Macho & Hembra & \\
\hline & CI & Promedio & 2 & 2 & 2 & 3 & 14 & 32 & 41 & 24 \\
\hline & Gl & Rang & $1.5-2$ & $1.5-2$ & $1.5-2$ & $3-4$ & $10-18$ & $29-36$ & $37-45$ & $18.5-29$ \\
\hline & & Promedio & 2 & 2 & 2 & 3 & 13 & 31 & 39 & 23 \\
\hline no & GII & Rango & $1.5-2$ & $1.5-2$ & $1.5-2$ & $3-4$ & $9-17$ & $28-35$ & $36-42$ & $17.5-28$ \\
\hline se & GIII & $\begin{array}{l}\text { Promedio } \\
\text { Rango }\end{array}$ & $\begin{array}{c}2 \\
1.5-2\end{array}$ & $\begin{array}{c}2 \\
1.5-2\end{array}$ & $\begin{array}{c}2 \\
1.5-2\end{array}$ & $\begin{array}{c}3 \\
3-4\end{array}$ & $\begin{array}{c}11 \\
8-17\end{array}$ & $\begin{array}{c}30 \\
26-34\end{array}$ & $\begin{array}{c}41 \\
38-45\end{array}$ & $\begin{array}{c}21 \\
16.5-28\end{array}$ \\
\hline
\end{tabular}
especies de parasitoides: Opius sp. (Hym: Braconidae), Halticoptera sp. (Hym: Pteromalidae), Ganaspidium sp. (Hym: Eucoilidae) y Chrysocharis sp. (Hym: Eulophidae). Estas especies son endoparasitoides, larva-pupa o larvopupales, los cuales fueron registrados para L. huidobrensis por Cisneros (1995), Sánchez \& Redolfi (1985) y Redolfi et al. (1988).

Ciclo biológico.

El ciclo biológico de $A$. maculosa, en alcachofa en la primera generación a temperatura de $24 \pm 1^{\circ} \mathrm{C}$ y $88 \pm 2 \%$ de humedad relativa fue en promedio 24 días,

Tabla 4. Proporción de sexos de Amauromyza maculosa Malloch (Dip.: Agromyzidae) en girasol bajo condiciones de laboratorio en tres generaciones. La Molina, Lima-Perú, 2006-2007.

\begin{tabular}{lcccccc}
\hline \multirow{2}{*}{ Generación } & \multirow{2}{*}{$\begin{array}{c}\text { N}^{\mathrm{o}} \text { de } \\
\text { individuos }\end{array}$} & \multicolumn{2}{c}{ Hembra $(\mathrm{H})$} & \multicolumn{2}{c}{ Macho (M) } & Proporción \\
\cline { 3 - 6 } & & Número & Porcentaje & Número & Porcentaje & $(\mathrm{M}: \mathrm{H})$ \\
\hline GI & 25 & 10 & 40 & 15 & 60 & $1.5: 1.0$ \\
GII & 25 & 11 & 36 & 16 & 64 & $1.5: 1.0$ \\
GIII & 25 & 10 & 40 & 15 & 60 & $1.5: 1.0$ \\
\hline
\end{tabular}

fueron. GI: $24 \pm 1^{\circ} \mathrm{C}$ y $88 \pm 2 \%$; GII: $25 \pm 1^{\circ} \mathrm{C}$ y $91 \pm 2 \%$; y GIII: $26 \pm 1^{\circ} \mathrm{C}$ y $90 \pm 2 \%$.

La temperatura y humedad relativa promedio fueron: GI: $25 \pm 1^{\circ} \mathrm{C}$ y $91 \pm 2 \%$; GII: $26 \pm 1^{\circ} \mathrm{C}$ y $90 \pm 2 \%$; y GIII: $25 \pm 2^{\circ} \mathrm{C}$ y $70 \pm 10 \%$.

\section{Conclusiones.}

Biología.

En alcachofa, el ciclo biológico es de 22.6 días; el huevo tiene un período de incubación de 2 días; la larva pasa por tres estadíos larvales, el periodo larval del primer $\mathrm{y}$ segundo estadío es de 2 días cada uno y el tercer estadío 3 días, con una duración larval promedio de 7 días; la pupa dura entre 11 a 14 días. El 
Tabla 6. Promedio en días de los estados de desarrollo de Amauromyza maculosa Malloch (Dip.: Agromyzidae), en girasol bajo condiciones de laboratorio en tres generaciones. La Molina, Lima-Perú. 2006-2007.

\begin{tabular}{|c|c|c|c|c|c|c|c|c|c|}
\hline \multirow{2}{*}{\multicolumn{2}{|c|}{ Generación }} & \multirow{3}{*}{$\begin{array}{c}\text { Huevo } \\
2\end{array}$} & \multicolumn{3}{|c|}{ Estadíos Larvales } & \multirow{3}{*}{$\frac{\text { Pupa }}{12}$} & \multicolumn{2}{|c|}{ Longevidad } & \multirow{3}{*}{$\begin{array}{c}\text { Ciclo Biológico } \\
22\end{array}$} \\
\hline & & & \multirow{2}{*}{$\begin{array}{l}1 \\
2\end{array}$} & \multirow{2}{*}{$\begin{array}{l}2 \\
2\end{array}$} & \multirow{2}{*}{$\begin{array}{l}3 \\
3\end{array}$} & & \multirow{2}{*}{$\begin{array}{c}\text { Macho } \\
27\end{array}$} & \multirow{2}{*}{$\begin{array}{c}\text { Hembra } \\
39\end{array}$} & \\
\hline & Promedio & & & & & & & & \\
\hline G1 & Rango & $1.5-2$ & $1.5-2$ & $1.5-2$ & $3-4$ & $9-17$ & $23-31$ & $36-42$ & $17-28$ \\
\hline & Promedio & 2 & 2 & 2 & 3 & 11 & 25 & 39 & 21 \\
\hline GII & Rango & $1.5-2$ & $1.5-2$ & $1.5-2$ & $3-4$ & $9-15$ & $21-29$ & $35-41$ & $17.5-26$ \\
\hline GIII & $\begin{array}{l}\text { Promedio } \\
\text { Rango }\end{array}$ & $\begin{array}{c}1 \\
1-2\end{array}$ & $\begin{array}{c}1 \\
1-2\end{array}$ & $\begin{array}{c}1 \\
1-2\end{array}$ & $\begin{array}{c}2.5 \\
2-3\end{array}$ & $\begin{array}{c}10 \\
6-13\end{array}$ & $\begin{array}{c}21 \\
19-23\end{array}$ & $\begin{array}{c}36 \\
33-40\end{array}$ & 16.5 \\
\hline
\end{tabular}

período de oviposición es de 38.8 días y la capacidad de oviposición de 326.6 huevos; la proporción sexual (M:H) fue de 1.1: 1.5 .

En girasol, el ciclo biológico dura 19.8 días. El huevo tiene un período de incubación de 2 días en dos generaciones y de 1 día en la tercera generación; la larva pasa por tres estadios larvales, el periodo larval del primer y segundo estadío dura 2 días cada uno en dos generaciones y 1 día cada estadío larval en la tercera generación y el tercer estadío larval dura 3 días pero en la tercera generación dura 2.5 días; la pupa dura 10 a 12 días. La oviposición dura 35.7 días. La capacidad de oviposición es de 345.3 huevos y la proporción sexual (M:H) es de 1: 1.5 .

Comportamiento.

Los huevos son endofíticos y colocados individualmente muy juntos entre sí. Las larvas pueden generar minas lagunares encontrándose de 9 a 63 larvas por mina. La larva de último estadío corta la epidermis de la hoja y sale de la mina para empupar generalmente al pie de la planta, bajo el rastrojo o en el suelo hasta $3 \mathrm{~cm}$ de profundidad. Los adultos son de hábitos diurnos; se alimentan y escogen el lugar de oviposición por las mañanas; la emergencia de adultos ocurre mayormente entre las 6 a.m. y 11 a.m.

\section{Literatura citada.}

Castillo J. 2006. Las principales plagas de alcachofa en el Perú. En Arenagro: Revista Institucional de la Asociación de Agricultores Agroexportadores Propietarios de terrenos (APTCH) de Chavimochic. Año 2, No. 3. Pág. 11-15.

Cisneros F. 1995. Control de las plagas agrícolas. Universidad Nacional Agraria La Molina. Segunda Edición. Lima, Perú. 313 p.

Guzmán M. \& Vergara C. 1996. Ciclo biológico de Liriomyza huidobrensis (Blanchard) en la variedad “Canchán”. En: Informe de investigación del proyecto: Biological and selective chemical control of potatoes and sweet potatoes insect pests. Lima. Universidad Nacional Agraria la Molina y Centro Internacional de la Papa.
Herrera J. 1963. Problemas insectiles del cultivo de papa en el valle de Cañete. Revista Peruana de Entomología. 6:19 .

Jara P. \& Alvites S. 1980. La mosca minadora de la papa: $L$. huidobrensis (Blanchard), Diptera: Agromyzidae. Boletín técnico $\mathrm{N}^{\circ}$ 6. Estación Experimental San Camilo- Ica.10 p.

Korytkowski G. 1982. Contribución al Conocimiento de los Agromyzidae (Diptera: Acalyptratae) en el Perú. Tesis (Magíster Scientiae, en la especialidad de Entomología). Universidad Nacional Agraria La Molina, Programa Académico de Graduados. 237 pp.

Lizárraga A. 1989. Biología de “mosca minadora” Liriomyza huidobrensis Blanchard (Díptera. Agromyzidae). Tesis para optar el título de Biólogo de la Universidad Ricardo Palma. Facultad de Ciencias Biológicas. 50 p.

Palacios M. \& Cisneros F. 1980. Biología y comportamiento de la mosca minadora Liriomyza huidobrensis B. Resúmenes XXIII Convención Nacional de Entomología- Huacho. 62 p.

Prando H. \& Da Cruz, F. 1986. Aspectos do biología de Liriomyza huidobrensis (Blanchard, 1926) (Diptera, Agromyzidae) en laboratorio. An Sociedad Entomologia Brasil. 15 (1): 77-78 p.

Raven K. 1993. Diptera III Aschiza y Acalyptratae. Universidad Nacional Agraria La Molina. Departamento de Entomología y Fitopatología. Lima-Perú.124 p.

Redolfi I., Rodríguez C. \& Ascensos D. 1988. Los parasitoides de Liriomyza huidobrensis Blanchard en cultivos de papa y frijol en la Molina. En: Informe de investigación del proyecto: Biological and selective chemical control of potatoes and sweet potatoes insect pests. Lima. Universidad Nacional Agraria la Molina y Centro Internacional de la Papa.

Robles F. 2000. Alcachofa: Nueva alternativa para la agricultura peruana. Orientaciones técnicas para su cultivo, empaque, proceso y exportación. Editorial PROMPEX-CESEM. Lima-Perú. 44 p.

Sánchez G. \& Redolfi I. 1985. Parasitoides de Liriomyza huidobrensis y Scrobipalpula absoluta en papa cultivada en Lima. Revista Peruana de Entomología. 28:81-84.

Siegel S. 1982. Estadística no paramétrica aplicada a las ciencias de la conducta. Séptima edición. Editorial Trillas. México. 344p.

\footnotetext{
${ }^{1}$ Tesis, Magister Scientiae. luenal20@hotmail.com

2 Docente de la Universidad Nacional Agraria La Molina, Av. La Molina s/n. Lima - Perú. joracava@lamolina.edu.pe

${ }^{3}$ Docente de la Universidad Nacional Agraria La Molina. srodriguez@lamolina.edu.pe
} 